\title{
SALIVARY MATRIX METALLOPROTEINASES ACTIVITY LEVELS IN AUTOIMMUNE DISEASES

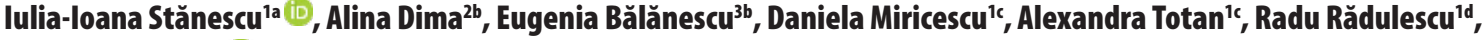

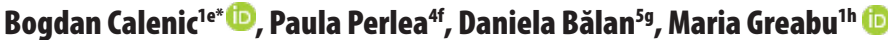

'Department of Biochemistry, Faculty of Dental Medicine, "Carol Davila” University of Medicine and Pharmacy, R0-050474 Bucharest, Romania 2Department of Internal Medicine, Faculty of Medicine, "Carol Davila"University of Medicine and Pharmacy, R0-020125 Bucharest, Romania ${ }^{3}$ Laboratory of Clinical Immunology, Colentina Clinical Hospital, R0-020125 Bucharest, Romania ${ }^{4}$ Department of Endodontics, Faculty of Dental Medicine, "Carol Davila" University of Medicine and Pharmacy, R0-010221 Bucharest, Romania 5Department of Physiology, Faculty of Dental Medicine, "Carol Davila” University of Medicine and Pharmacy, RO-010221 Bucharest, Romania

\author{
aDDS, Assistant Professor \\ ${ }^{\mathrm{b} M D,}$ PhD \\ PhD, Assistant Professor \\ ${ }^{\mathrm{D}} \mathrm{DDS}, \mathrm{PhD}$ \\ eDDS, PhD, Assistant Professor \\ 'DDS, PhD, Professor \\ 9MD, PhD, Assistant Professor \\ hPhD, Professor, Head
}

Introduction: It is widely recognized that saliva represents a solid alternative as a diagnostic fluid in a wide range of oral and general diseases. Autoimmune diseases constitute an important health threat to both men and women worldwide with matrix metalloproteinases (MMPs) playing significant roles in pathogenesis and development of these diseases. The specific aim of the present study is to asses, for the first time, salivary levels of a selected panel of MMPs in several autoimmune diseases.

Methodology: The study included 30 patients divided into groups such as: systemic sclerosis, vasculitis and healthy subjects. Salivary levels of MMP-1, $-2,-7,-9$ and -10 were analyzed using magnetic bead-based multiplex assays and Luminex technology.

Results: MMP-2 salivary levels were statistically elevated in systemic sclerosis, while MMP-10 were also increased in vasculitis patients. Salivary levels of MMP-9 were found significantly increased in all analyzed groups.

Conclusion: Taken together, our results promote saliva as a reliable diagnostic fluid for quantifying MMPs in autoimmune diseases.

Keywords: saliva, autoimmune diseases, matrixmetalloproteinase, vasculitis, systemic sclerosis.

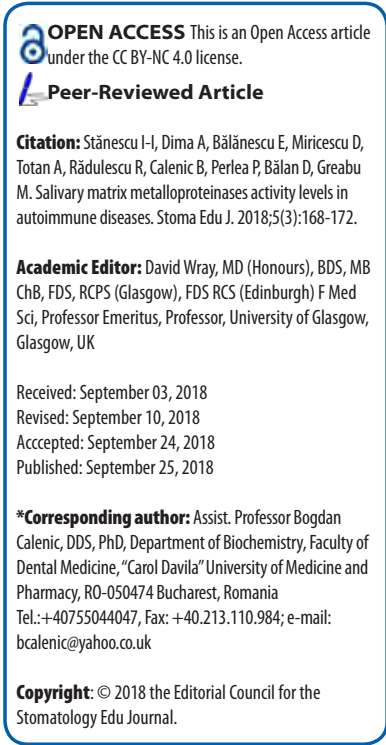

\section{Introduction}

Over the past decades, saliva has become a hot topic in medical research related to discovering alternative diagnostic methods. Whole saliva is a complex fusion of the fluid produced by major and minor salivary glands with gingival crevicular fluid, oral desquamated cells, bacteria, as well as with food remains $[1,2]$. The advantages provided by saliva as a diagnostic fluid include non-invasive and effortless collection procedures, the need for small samples for analysis, along with easy storage and an established correlation between salivary and blood biomarkers' levels [3]. In addition to these benefits, its rich composition consisting of electrolytes, proteins and hormones deriving not only from oral sources, but also from systemic production, promote saliva as a remarkable diagnosis and monitoring fluid for both local and general diseases [4-6]. However, few studies have focused on the potential saliva has as a diagnostic fluid for autoimmune diseases $[7,8]$.
Scleroderma is considered a rare disease that affects the connective tissue. Depending on the extent of the manifestations, scleroderma can be classified either as localized- when only the skin and in some cases the tissues underneath are involved, or systemic sclerosis, a more complex form in which cutaneous manifestations are associated with visceral impairment. Theories regarding the etiology of this affliction have incriminated genetic factors, trauma, viral or bacterial infections, neurological damage or vascular abnormalities. However, an increased level of antinuclear antibodies, as well as the frequent association with autoimmune diseases such as systemic vasculitis or systemic sclerosis, plead for an autoimmune disease characterized by an inflammatory phase followed by fibrosis, causing atypical collagen to replace normal dermis [9]. Vasculitis is a rare disease characterized by an inflammatory process located within the walls of blood vessels, affecting mainly small calibre vessels. The great diversity of the vessels affected 
explains the clinical heterogeneity of this disease that involves a great variety of organs [10]. Meanwhile, matrix metalloproteinases (MMPs) represent a group of proteases produced by several types of cells, including pro-inflammatory cells with multiple roles such as leukocyte infiltration in conditions with an inflammatory component [11]. To the best of our knowledge up to the present, there are no scientific studies focusing on the salivary MMP levels in patients with autoimmune diseases.

Taking all this into consideration, the main objective of the present research is to assess the potential use of saliva as a diagnosis and monitoring fluid for autoimmune diseases by evaluating salivary levels of a group of MMPs in patients with systemic sclerosis and vasculitis and compare them with those of healthy subjects.

\section{Material and methods}

\subsection{Patient selection}

Our study included a total of 30 patients: 10 patients with systemic sclerosis and 10 vasculitis patients. 10 healthy subjects, with no autoimmune disease, represented the control group. Clinical data including age, gender, current treatments, as well as a series of biochemical and immunological parameters have been collected for all the participants to the study. All subjects included in our research agreed to participate voluntarily and signed an informed consent. The present research was approved by the ethics board of Colentina Clinical Hospital, Bucharest, Romania. All patients were diagnosed by the same experienced medical professional.

\subsection{Samples collection}

For all participants in the study, saliva samples were collected at the moment of inclusion in the present study. Their written consent was taken prior to collecting the samples. The collection method followed a well-established protocol and took place as follows: $2-5 \mathrm{~mL}$ of unstimulated whole saliva was given by each participant in the morning around 9 AM. The participants were asked to refrain from eating, drinking or smoking prior to saliva collection. All subjects rinsed with $5 \mathrm{~mL}$ of distilled water before collection. The patients were also asked to sit down and be relaxed during saliva sampling as well as not to communicate with each other. Immediately after the collection, the samples were kept on ice and transported in an isotherm box and subsequently, they were centrifuged at $5000 \mathrm{rpm}$ for 10 minutes. Samples were aliquoted and stored at $-80^{\circ} \mathrm{C}$ until further determinations.

\subsection{Matrix metalloproteinases detection}

All MMPs were detected using Luminex technology and Magnetic Bead-Based Multiplex assays. This method enables the simultaneous detection of multiple salivary human biomarkers. Thus salivary levels of MMP-1, -2, $-7,-9,-10$ were assessed using MILLIPLEX MAP Human MMP Magnetic Bead Panel 2 - Immunology Multiplex Assay. All samples and controls were processed following the manufacturer's specifications. The assay required $25 \mu \mathrm{L}$ of saliva diluted at 1:20; each MMP had its own detection interval as follows: MMP-1: 27 -
I Table 1. Clinical data of patients included in the study (autoimmune disease group and control group).

\begin{tabular}{|c|c|c|c|c|}
\hline & Cases & $\begin{array}{c}\text { Inclusion } \\
\text { age, } \\
\text { Years }\end{array}$ & $\begin{array}{c}\text { Disease } \\
\text { duration, } \\
\text { Years }\end{array}$ & $\begin{array}{l}\text { BMI, } \\
\mathrm{kg} / \mathrm{m}^{2}\end{array}$ \\
\hline $\begin{array}{c}\text { Total } \\
\text { subjects }\end{array}$ & 63 & $\begin{array}{c}53.0 \\
(37.5-60.5)\end{array}$ & $\begin{array}{c}8.0 \\
(4.0-14.0)\end{array}$ & $\begin{array}{c}25.5 \\
(21.8-29.9)\end{array}$ \\
\hline SS & 10 & $\begin{array}{c}52.0 \\
(40.0-60.0)\end{array}$ & $\begin{array}{c}2.0 \\
(0.5-9.0)\end{array}$ & $\begin{array}{c}25.9 \\
(22.4-30.9)\end{array}$ \\
\hline VA & 10 & $\begin{array}{c}47.0 \\
(42.0-70.0)\end{array}$ & $\begin{array}{c}8.0 \\
(2.0-14.0)\end{array}$ & $\begin{array}{c}25.8 \\
(24.8-35.1)\end{array}$ \\
\hline Control & 10 & $\begin{array}{c}45.0 \\
(30.0-50.5)\end{array}$ & - & $\begin{array}{c}21.9 \\
(19.5-22.6)\end{array}$ \\
\hline
\end{tabular}

SS-systemic sclerosis; VA -vasculitis; BMI-body mass index; kg-kilogram; $m$-meter,

Table 2.MMP levels in Systemic Sclerosis. SS n-10 cases.

\begin{tabular}{cccccc}
\hline & MMP1 & MMP2 * & MMP7 & MMP9 * & MMP10 * \\
\hline \multirow{4}{*}{ SS } & 1424.43 & 24.3125 & 1093.68 & 6571.25 & 1796.62 \\
& $(1009.22-$ & $(19.32-$ & $(1003.02-$ & $(5894.31-$ & $(1221.33-$ \\
& $1994.30)$ & $29.33)$ & $1903.10)$ & $7043.44)$ & $1943.01)$ \\
& & & & & \\
Control & 3565.167 & 10.3 & 1521.45 & 1148.5 & 943.82 \\
& $(1237.5-$ & $(3.45-$ & $(854.3-$ & $(843.4-$ & $(754.28-$ \\
& $4422.5)$ & $14.32)$ & $2321.59)$ & $2749.48)$ & $1232.37)$
\end{tabular}

Data are expressed as median (inferior; superior limits); ${ }^{*}$ Mann-Whitney test; $p$-value $<0.05$ is considered statistically significant

Table 3. MMP levels in Vasculitis. VA n-10 cases.

\begin{tabular}{cccccc}
\hline & MMP1 & MMP2 & MMP7 & MMP9 * & MMP10 * \\
\hline \multirow{4}{*}{ VA } & 1899.7 & 25.4 & 1071 & 5551.7 & 2372.2 \\
& $(1002.54-$ & $(10.22-$ & $(920.32-$ & $(4988.38-$ & $(2082.10-$ \\
& $2540.9)$ & $38.43)$ & $1643.39)$ & $5903.80)$ & $3002.48)$ \\
\multirow{4}{*}{ Control } & 3565.167 & 10.3 & 1521.45 & 1148.5 & 943.82 \\
& $(1237.5-$ & $(3.45-$ & $(854.3-$ & $(843.4-$ & $(754.28-$ \\
& $4422.5)$ & $14.32)$ & $2321.59)$ & $2749.48)$ & $1232.37)$
\end{tabular}

Data are expressed as median (inferior; superior limits); ${ }^{*}$ Mann-Whitney test; $p$-value $<0.05$ is considered statistically significant

20,000 pg/mL; MMP-2: 68 - 50,000 pg/mL; MMP-7: 548 - 400,000 pg/mL; MMP-9: 14 - 10,000 pg/mL; MMP10: $27-20,000 \mathrm{pg} / \mathrm{mL}$. The assay involved a two-hour multistep procedure involving preparation of reagents, samples and controls, preparation of the 96 well-plate and analysis using a Luminex ${ }^{\circledR} 200^{\mathrm{TM}}$ and the xPONENT ${ }^{\circledR}$ software for data acquisition and analysis.

\subsection{Statistical analysis}

The statistical analysis was performed using SPSS software. The characteristics were expressed as median (quartile 1; 3). Statistically significant correlations were found using the Mann-Whitney test (a twosided $p$-value less than 0.05 was noted as statistically significant). A Spearman test was used to evaluate possible correlations between salivary MMPs levels, as well as for other significant bivariate ( $p$-value $<0.05$ was considered statistically significant).

\section{Results \\ 3.1. Clinical data}

Several clinical parameters such as body mass index, age at the moment of the diagnostic and at the inclusion as well as disease duration were recorded for all subjects included in the study (see Table 1). 


\subsection{MMP-1, -2, -7, -9, 10 levels in Systemic Sclerosis} In the present study patients with SS showed a statistical increment in salivary levels of MMP-2, -9 and -10 when compared with respective controls. MMP-7 was also increased, but with no statistical significance. MMP-1 were statistically lower in the SS group vs the control group (Table 2).

\subsection{MMP-1, -2, -7, -9, 10 levels in Vasculitis}

Patients suffering from vasculitis had statistically increased levels of MMP-9 and -10. At the same time, the data were not statistically relevant for MMP-1, -2 and MMP-7 (Table 3).

\section{Discussion}

In the past, blood serum has routinely been used in the diagnostic process. However, most of the blood constituents are found in the saliva, as they pass through transcellular and paracellular routes. Nowadays saliva could be introduced as a diagnostic tool in many medical fields including microbiology, immunology, oncology, endocrinology, etc. Saliva is easy to collect, ship and store; it could be obtained in sufficient quantities at a low cost via non-invasive methods $[12,13]$. Saliva-testing kits have been presented on the market, although some of them are still waiting for approval. Intense efforts have been undertaken to detect markers that reflect tumor pathologies such as oral cancers. The composition of saliva also reflects pathological changes in salivary glands. Salivary tumors can release in the salivary flow important levels of stathmin or maspin, tumor necrosis factor, Dim1p, v-Ha-ras oncogene, type I collagen pro alpha or pirin [14]. Systemic cancers such as breast, gastric or larynx neoplasm can also be detected in saliva. $p 53$ is a tumor suppressor protein capable of blocking the tumor formation. Mutations in $p 53$ gene are found in most of the tumors and are associated with tumor development and poor prognosis. p53 antibodies are found in the serum and saliva of patients with oral squamous cell carcinoma [15]. Another marker for oral squamous cell carcinoma identification, with significantly increased levels in saliva, is cortisol. The marker is even frequently used for tumor staging. Some growth factors concentrations in saliva also correlate with the development of neoplasms. Elevated levels of Fibroblast growth factor 2 (FGF2) correspond to salivary gland tumor growth. Sjögren syndrome, an autoimmune disease which among other effects alters the composition of saliva and tears is also studied in association with reactive species of oxygen (ROS) changes [16]. Several recent studies have shown that the biomarker composition is different in saliva from patients with Sjögren syndrome versus controls. Other studies analyzed saliva in connection with diseases such as Alzheimer, anorexia, bulimia of mental stress [17].

MMP-1 is a collagenase that normally can be found in low quantities in the cells, but increases its levels in autoimmune diseases as well as in inflammation. This protease that degrades collagen plays an important role in wound healing, immune response, inflammation, fibrotic disorders and cardiovascular diseases. Moreover, MMP-1 could be involved in cancer. Our data did not show an increased activity of MMP-1 in any included
Table 4. Increased salivary MMPs levels in autoimmune diseases (increments are based on own results and are statistically significant at a $p$ $<0.05)$.

\begin{tabular}{ccc}
\hline & SS & VA \\
\hline MMP-1 & - & - \\
MMP-2 & $\uparrow 2$ fold & - \\
MMP-7 & - & - \\
MMP-9 & $\uparrow 6$ fold & $\uparrow 4$ fold \\
MMP-10 & $\uparrow 2$ fold & $\uparrow 2$ fold \\
\hline
\end{tabular}

autoimmune disease. Moreover, salivary MMP-1 was decreased SS and VA when compared to their controls, but the results were not statistically significant. These findings corroborated with existing scientific literature may suggest that MMP-1 does not play an active role in the pathogenesis of autoimmune diseases. Considering their organization, MMP-2 and MMP-9 are considered gelatinases. MMP-2 plays important roles in both physiological processes such as angiogenesis and in pathological situations like varicose veins disease, inflammation, and cardiovascular diseases. Meanwhile, MMP-9 in involved in cell apoptosis and osteoarthritis. MMP-9 was shown to present higher levels in patients with systemic sclerosis [18]. Interestingly, in our study salivary MMP-9 levels were found statistically higher in all patient groups included in the study when compared to the respective controls. Knowing that MMP-9 is generally involved in programmed cell death we can infer that this marker is involved in tissue remodeling processes occurring in these diseases. MMP-7 belongs to the matrilysins subgroup and has been linked to inflammation, lung disease, cardiovascular afflictions and to physiological cell apoptosis. In the present study, MMP-7 salivary levels were not statistically elevated in any studied disease. MMP- 10 or stromelysin 2 has been linked to liver disease, viral infections, lung disease, tissue remodeling and cell apoptosis and is considered to play an important role in peripheral arterial disease. Our findings show that MMP-10 salivary levels were significantly elevated when compared to controls in both systemic sclerosis and vasculitis patients.

The present study has several limitations that will be addressed in further studies: a larger number of patients will be needed in the future to validate the present findings. Future correlations with MMP serum levels in all patients groups are needed in order to address the possible utility of salivary MMPs in clinical settings.

\section{Conclusion}

In conclusion, the present study shows for the first time, that important matrix metalloproteinase is increased and can be detected in saliva from patients with autoimmune diseases. Thus, salivary determination of MMP-1, $-2,-7,-9$ and -10 promotes saliva as a viable alternative when determining the activity of these enzymes in systemic sclerosis or vasculitis.

\section{Conflicts of Interest}

The authors declare no conflict of interest. 


\section{Author Contributions}

All authors equally contributed to the present manuscript. IIS: participated in study design, data collection, data interpretation and manuscript writing; AD: participated in study design and sample collection; EB: participated in sample collection; DM: participated in sample analysis and manuscript writing; AT: participated in manuscript writing; RR: participated in data collection, data interpretation; BC: participated in study design, data collection, data interpretation and manuscript writing; PP: participated in critical review of the manuscript; DB: participated in critical review of the manuscript; MG: participated in study design, data collection, data interpretation and manuscript writing.

\section{References}

1. Castagnola MP, Picciotti PM, Messana I, et al. Potentia applications of human saliva as diagnostic fluid. Acta Otorhinolaryngol Ital. 2011;31(6):347-357.

[Free PMC Article] [PubMed] Google Scholar (131) Scopus (68)

2. Nunes LA, Mussavira S, Bindhu OS. Clinical and diagnostic utility of saliva as a non-invasive diagnostic fluid: a systematic review. Biochem Med. (Zagreb). 2015;25(2):177-192. doi: 10.11613/ BM.2015.018. eCollection 2015.

[Full text links] [Free PMC Article] [PubMed] Google Scholar (90) Scopus (59)

3. Yoshizawa JM, Schafer CA, Schafer JJ, et al. Salivary biomarkers: toward future clinical and diagnostic utilities. Clin Microbiol Rev. 2013;26(4):781-791. doi: 10.1128/CMR.00021-13.

[Full text links] [Free PMC Article] [PubMed] Google Scholar (155) Scopus (96)

4. Stănescu II, Calenic, B, Dima A, et al. Saliva as a monitoring fluid for hormonal activity in systemic lupus erythematosus. REV CHIM. (Bucharest). 2018;69(3):654-659.

Google Scholar (0) Scopus (0)

5. Stănescu II, Calenic B, Dima A, et al. Salivary biomarkers of inflammation in systemic lupus erythematosus. Ann Anat. 2018;219:89-93. doi: 10.1016/j.aanat.2018.02.012.

[Full text links] [PubMed] Google Scholar (0) Scopus (0)

6. Pfaffe T, Cooper-White J, Beyerlein P, et al. Diagnostic potential of saliva: current state and future applications. Clin Chem. 2011;57(5):675-687. doi: 10.1373/clinchem.2010.153767. [Full text links] [PubMed] Google Scholar (406) Scopus (257)

7. Hu S, Vissink A, Arellano M, et al. Identification of autoantibody biomarkers for primary Sjögren's syndrome using protein microarrays. Proteomics. 2011;11(8):1499-1507. doi: 10.1002/ pmic.201000206.

[Full text links] [Free PMC Article] [PubMed] Google Scholar (80) Scopus (59)
8. Bonamico M, Nenna R, Montuori M, et al. First salivary screening of celiac disease by detection of anti-transglutaminase autoantibody radioimmunoassay in 5000 Italian primary schoolchildren. J Pediatr Gastroenterol Nutr. 2011;52(1):17-20. doi: 10.1097/MPG.0b013e3181e6f2d0.

[Full text links] [PubMed] Google Scholar (53) Scopus (29)

9. Careta MF, Romiti R. Localized scleroderma: clinical spectrum and therapeutic update An Bras Dermatol. 2015;90(1):62-73. doi: 10.1590/abd1806-4841.20152890.

[Full text links] [Free PMC Article] [PubMed] Google Scholar (43) Scopus (25)

10. McKinney EF, Willcocks LC, Broecker V, Smith KG. The immunopathology of ANCA-associated vasculitis. Semin Immunopathol. 2014;36(4):461-478. doi: 10.1007/s00281-0140436-6.

[Full text links] [Free PMC Article] [PubMed] Google Scholar (28) Scopus (17)

11. Cui N, Hu M, Khalil RA. Biochemical and biological attributes of matrix metalloproteinases. Prog Mol Biol TransI Sci. 2017;147:173. doi: 10.1016/bs.pmbts.2017.02.005.

[Full text links] [Free PMC Article] [PubMed] Google Scholar (23) Scopus (14)

12. Dawes $C$, Pedersen $A M$, Villa $A$, et al. The functions of human saliva: A review sponsored by the World Workshop on Oral Medicine VI. Arch Oral Biol. 2015J;60(6):863-874. doi: 10.1016/j. archoralbio.2015.03.004.

[Full text links] [PubMed] Google Scholar (118) Scopus (70)

13. Javaid MA, Ahmed AS, Durand R, Tran SD. Saliva as a diagnostic tool for oral and systemic diseases. J Oral Biol Craniofac Res. 2016;6(1):66-75. doi: 10.1016/j.jobcr.2015.08.006.

[Full text links] [Free PMC Article] [PubMed] Google Scholar (61) Scopus (37)

14. Cheng YS, Rees T, Wright J. A review of research on salivary biomarkers for oral cancer detection. Clin Transl Med. 2014:3(1):3. doi: 10.1186/2001-1326-3-3.

[Full text links] [PubMed] Google Scholar (110)

15. Aoyama I, Yaegaki K, Calenic B, et al. The role of $\mathrm{p} 53$ in an apoptotic process caused by an oral malodorous compound in periodontal tissues: a review. J. Breath Res. 2012:6(1):017104. doi: 10.1088/1752-7155/6/1/017104.

[Full text links] [PubMed] Google Scholar (14) Scopus (7)

16. Wang J, Schipper HM, Velly AM, et al. Salivary biomarkers of oxidative stress: A critical review. Free Radic Biol Med. 2015;85:95-104. doi: 10.1016/j.freeradbiomed.2015.04.005. [Full text links] [PubMed] Google Scholar (35) Scopus (20)

17. Malathi N, Mythili S, Vasanthi HR. Salivary diagnostics: a brief review. ISRN Dent. 2014;2014:158786. doi: 10.1155/2014/158786. eCollection 2014

[Full text links] [Free PMC Article] [PubMed] Google Scholar (65)

18. Kim WU, Min SY, Cho ML, et al. Elevated matrix metalloproteinase-9 in patients with systemic sclerosis. Arthritis Res Ther. 2005;7(1):R71-9. doi: 10.1186/ar1454.

[Full text links] [Free PMC Article] [PubMed] Google Scholar (86) Scopus (61)

\section{Iulia-Ioana STĂNESCU \\ DDS, Assistant Lecturer Department of Biochemistry, Faculty of Dental Medicine "Carol Davila" University of Medicine and Pharmacy Bucharest, Romania}

Iulia-loana Stănescu is a dentist, currently a PhD candidate in the field of Biochemistry and an Assistant Lecturer in the Department of Physiology at the Faculty of Dental Medicine, "Carol Davila" University of Medicine and Pharmacy, Bucharest Romania. She graduated from the Faculty of Dental Medicine, "Carol Davila" University of Medicine and Pharmacy in dentistry and her field of interest in her research as a PhD candidate is salivary diagnosis in autoimmune diseases. 


\section{Ouestions}

\section{Saliva is:}

口a. A reliable diagnostic fluid containing biomarkers that statistically correlate with those found in blood; b. Not a reliable diagnostic fluid containing biomarkers that statistically correlate with those found in blood;

Dc. A fluid produced by major and minor salivary glands with gingival crevicular fluid, oral desquamated cells, but not oral bacteria;

$\square$ d. An invasive method of collection.

\section{Vasculitis:}

$\square$ a. Is a common autoimmune disease;

b. Usually affects large blood vessels;

ac. Can affect only a few organs;

ad. Is located in the walls of blood vessels.

\section{MMP-2 is:}

Da. Increased 2 fold in saliva from patients with vasculitis;

b. Increased 2 fold in saliva from patients with systemic sclerosis;

uc. Not statistically increased in saliva samples taken from patients with autoimune diseases;

$\square d$. Not a gelatinase.

\section{MMP-9 is:}

Da. Increased 6 fold in saliva from patients with systemic sclerosis;

b. Increased 2 fold in in saliva from patients with systemic sclerosis;

口c. Statistically decreased in saliva samples taken from patients with autoimune diseases;

$\square d$. Not involved in apoptosis.

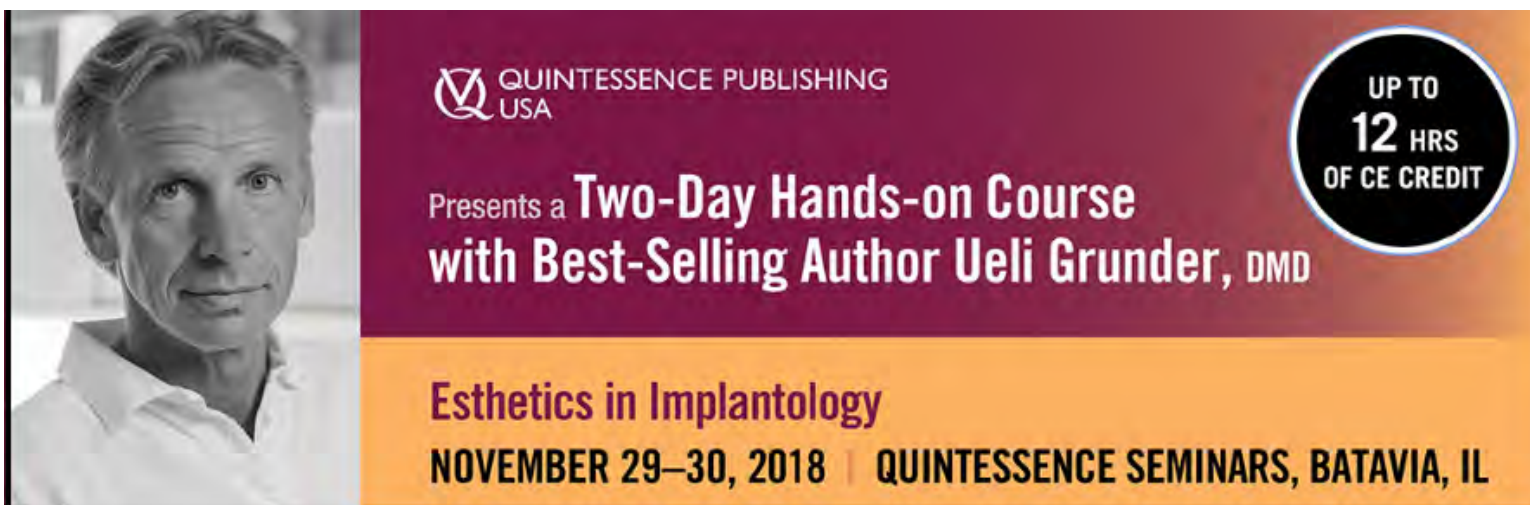

http://www.quintpub.com/grunder/ 